rasosem

\title{
Evaluación de la implementación de un Programa de Uso Optimizado de Antimicrobianos en la pandemia de COVID-19
}

\author{
Recibido: 29/10/20 Aceptado: 10/11/20
}

Laura Jorge ${ }^{1}$, María Cecilia Lauricella², Diana Silvia Klajn ${ }^{3}$, Sofía del Valle ${ }^{4}$, Ana María Molina², Adriana Cagnoni², Diego Russo Lacerna ${ }^{2}$, Regina Spiratos ${ }^{2}$ y Viviana M. Rodríguez ${ }^{2}$.

\section{RESUMEN}

Introducción: No obstante la baja frecuencia de infecciones bacterianas asociadas al COVID-19, la prevalencia del uso de antibióticos empíricos es de 70 a $90 \%$. El objetivo primario del estudio fue evaluar el impacto de la implementación de un programa de optimización de antimicrobianos dirigido a pacientes con COVID-19 (PROA-COVID).

Material y métodos: Estudio antes y después, retrospectivo, descriptivo y analítico. Se evaluó prevalencia y adecuación del uso de antibióticos antes y después de implementación del PROA-COVID en pacientes internados. Se estimó consumo mensual de antibióticos en DDD/100 pacientes-día y costos por uso inadecuado.

Resultados: Se incluyeron 153 pacientes, 75 antes y 78 después de la intervención, sin diferencias significativas en las características poblacionales entre ambos períodos. Las formas clínicas moderadas-severas fueron más frecuentes postintervención $(p=0,03)$. La implementación mostró una disminución significativa en prevalencia de uso $(64 \%$ vs $41 \%, p=0,004)$, con aumento de uso adecuado $(37,5 \%$ vs. $46,8 \%, p=\mathrm{NS})$. La indicación innecesaria fue mayor antes del PROA ( $80 \%$ vs $50 \%, p=0,03)$ y la duración del tratamiento postintervención ( $13,3 \%$ vs. $43,7 \% \%, p=0,02)$. La implementación redujo el consumo de betalactámicos + IBL y azitromicina.

No se observaron diferencias significativas en mortalidad, frecuencia de pase a UCC ni uso de antibioticoterapia combinada con macrólidos entre ambos períodos ajustando por severidad.

\begin{abstract}
${ }^{1}$ Programa de Optimización de Uso de Antimicrobianos, Ministerio de Salud de la Ciudad de Buenos Aires, Argentina.

${ }^{2}$ Servicio de Infectología Hospital General de Agudos Enrique Tornú, Buenos Aires, Argentina.

${ }^{3}$ Comité de Docencia e Investigación, Hospital General de Agudos Enrique Tornú, Buenos Aires, Argentina.

${ }^{4}$ División Farmacia, Hospital General de Agudos Enrique Tornú, Buenos Aires, Argentina.

Dirección para la correspondencia: Laura Jorge, Combatientes de Malvinas 3002 - CABA - (CP 1427) Email: laurajorge1981@gmail.com

Los contenidos del material a ser evaluado no han sido publicados anteriormente. No cuenta con fuentes de apoyo. Ninguno de los autores presenta conflicto de intereses en relación a esta publicación.
\end{abstract}

Conclusiones: La implementación del PROA-COVID fue una estrategia efectiva para reducir el uso de antibióticos y optimizar sus indicaciones, lo que destaca la importancia de su aplicación rápida y oportuna.

Palabras clave: Programa de Uso Optimizado de Antimicrobianos, COVID-19, antibiótico. 


\section{Introducción}

La emergencia del SARS-CoV-2 y la enfermedad por coronavirus 2019 (COVID-19) en diciembre de 2019 ha sido un gran desafío para el sistema de salud. Las decisiones que conciernen a la atención de los pacientes son tomadas con limitada experiencia clínica y evidencia científica. Una de estas decisiones es el uso de antibióticos (ATB) en pacientes con COVID-19, donde la racionalidad para el mismo está basada en la experiencia previa de coinfecciones en pacientes con influenza $(1,2)$, en los que está descrito hasta un $30 \%$ de coinfecciones bacterianas. Estas no están descritas en la infección por MERS-CoV y ocurren raramente en pacientes con SARS-CoV1 (3).

La evidencia disponible hasta el momento muestra que las coinfecciones bacterianas en pacientes con COVID-19 son muy poco frecuentes. Un meta-análisis describe que solo el $7 \%$ de los pacientes hospitalizados presentan coinfección bacteriana (4), al igual que otro estudio que describe una prevalencia del $8 \%$ (5). Sin embargo, los mismos estudios muestran que el uso de antibióticos empíricos asciende hasta el 70 a $90 \%$ de los pacientes ingresados, y que la mayoría recibe antibióticos de amplio espectro (4-6).

En coincidencia con este incremento del consumo de ATB comenzó a describirse un aumento de las infecciones intrahospitalarias y una mayor incidencia de microorganismos multirresistentes (OMR) (7-9).

Todo esto remarca la importancia de la aplicación y adecuación de los PROA en estos tiempos tan desafiantes.

Las guías de tratamiento disponibles no recomiendan el uso de ATB de rutina en pacientes con COVID-19 leves y moderados. El tratamiento antibiótico empírico está indicado en pacientes con COVID-19 severo y crítico o en pacientes con enfermedad moderada que presentan características de infección bacteriana, como hallazgos focales en Rx o TC de tórax o aumento del recuento de neutrófilos $(10,11)$.

Es previsible que con el avance de la epidemia un mayor número de pacientes reciba terapia antimicrobiana empírica. El uso inadecuado de antibióticos tiene consecuencias en el corto y largo plazo que incluyen toxicidades, infecciones por C. difficile y la emergencia y propagación de la resistencia antimicrobiana (RAM) $(12,13)$.

El objetivo primario del estudio fue evaluar el impacto de la implementación de un PROA dirigido al uso de antimicrobianos en pacientes con COVID-19 sobre el consumo y adecuación de su indicación. Su objetivo secundario fue estimar el ahorro en los costos de los tratamientos inadecuados evitados con la implementación de dicho programa.

\section{Materiales y métodos}

Se realizó un estudio antes y después/serie de tiempo interrumpida, retrospectivo, descriptivo y analítico. Se incluyeron pacientes mayores de 16 años que ingresaron a las salas generales del hospital como casos confirmados de COVID-19 entre mayo y septiembre de 2020. Se excluyeron aquellos que ingresaron a las salas generales de internación del hospital como casos confirmados de COVID-19 y que presentaron otro foco infeccioso, por lo cual requirieron tratamiento antibiótico, quienes fueron derivados desde otros centros o desde las unidades de terapia intensiva luego de un período mayor a 48 horas desde el diagnóstico y los pacientes que ingresaron al hospital con otros diagnósticos y que durante la internación adquirieron COVID-19.

La intervención consistió en la implementación de un Programa de Uso Optimizado de Antimicrobianos dirigido a pacientes con diagnóstico de COVID-19 (PROA-COVID), para lo que se generó una norma específica que se difundió como un documento y se presentó en el ateneo central del servicio de Clínica Médica. En la misma se definió la indicación adecuada de ATB en función de lo sugerido en guías internacionales de tratamiento COVID-19 $(10,11)$, donde se indicó que el uso de ATB estuviera restringido a determinados criterios: i) pacientes con COVID-19 severo y ii) pacientes con COVID-19 moderado que tuvieran sospecha de infección bacteriana (laboratorio con leucocitosis o radiografía o tomografía de tórax con infiltrados focales). En el caso de utilizar ATB, las opciones sugeridas fueron: amoxicilina-clavulánico, ampicilinasulbactam, ceftriaxona, levofloxacina y claritromicina. Se sugirió el tratamiento combinado con macrólidos para los casos de neumonías severas. La duración del tratamiento recomendada fue de 5 a 7 días. Luego de la difusión inicial de la norma se continuaron realizando auditorías prospectivas para evaluar la adherencia a la misma.

Los pacientes que estuvieron internados en sala general desde 20 días antes de la aplicación del PROA dirigido a COVID-19 hasta su aplicación constituyeron el grupo previo al programa (P1). Como grupo posterior (P2) al programa se incluyeron todos los pacientes internados desde el mes de aplicación del PROA dirigido a COVID-19 hasta completar un número igual al del grupo P1. 
Se evaluaron comorbilidades (diabetes, hipertensión arterial, enfermedad pulmonar obstructiva crónica (EPOC), asma, obesidad, inmunosupresión activa), la duración de la internación, el requerimiento de pase a una unidad de cuidados críticos (UCC) y la mortalidad hospitalaria. Se definió la severidad de la enfermedad como: a) leve: presencia de signos y síntomas de COVID-19 sin neumonía, b) moderada: presencia de neumonía con saturación de oxígeno (Sat. $\mathrm{O}_{2}$ ) mayor o igual a $94 \%$ al aire ambiente y c) severa: frecuencia respiratoria > 30 respiraciones/min; Sat. $O_{2}<94 \%$; cociente presión de oxígeno en sangre/fracción inspirada de oxígeno $\left(\mathrm{PO}_{2} / \mathrm{FIO}_{2}\right)<300 \mathrm{mmHg}$ o infiltrados pulmonares $>50 \%$ (14).

Los hallazgos en la radiografía de tórax fueron clasificados como: a) hallazgos consistentes con COVID-19 (infiltrados difusos bilaterales) y b) infiltrados focales. Se utilizaron como fuente de información las historias clínicas y los informes del servicio de imágenes.

Además se evaluó el consumo mensual de antibióticos (ATB) en DDD/100 pacientes días y el exceso de costos calculados por el uso inadecuado de ATB durante el P1 y proyectados en el tiempo que duró el estudio.

Se utilizó estadística descriptiva para caracterizar la población de estudio. Para las variables continuas se utilizaron medidas de centralidad (media o mediana) y dispersión (desvío estándar o rango intercuartílico) adecuadas según la distribución de las variables. Para las variables categóricas se utilizaron frecuencias absolutas y relativas. Para el análisis estadístico se utilizó la prueba de chi-cuadrado, test exacto de Fisher, prueba T de Student o prueba de Mann Whitney según correspondiera. Se elaboraron distintos modelos de regresión con pase a UCC, mortalidad y tratamiento ATB combinado como variables de resultado y período como variable regresora, ajustado por aquellas que mostraron diferencias en el análisis bivariado. Todas las pruebas se realizaron con un nivel de significación de 0,05 y en todos los casos se comprobaron los supuestos que sustentan los tests. Se utilizó el software estadístico STATA 10.0.

El protocolo fue aprobado por el Comité de Ética en Investigación del Hospital Tornú.

\section{Resultados}

Se incluyeron 153 pacientes con COVID-19, 75 antes de la implementación del PROA y 78 luego de ella. Las características de los pacientes en ambos períodos se describen en la Tabla 1.

No se observaron diferencias estadísticamente significativas en edad, sexo ni comorbilidades entre ambos períodos.

En cuanto a la presentación clínica de COVID-19 en el primer período, predominaron las formas leves y moderadas $(29,3 \%$ y $45,3 \%)$ frente a las formas moderadas y graves $(51,3 \%$ y $33,3 \%)$ en el segundo período $(p=0,03)$.

\section{Tabla 1. Características generales de la población. Comparación de ambos períodos}

\begin{tabular}{|c|c|c|c|}
\hline & $\begin{array}{c}P 1 \\
n=75\end{array}$ & $\begin{array}{c}P 2 \\
n=78\end{array}$ & p-valor \\
\hline Sexo femenino, n (\%) & $37(49,3)$ & $36(46,1)$ & 0,694 \\
\hline Edad, promedio (DE) & $50.9(17,3)$ & $55.1(18,1)$ & 0,148 \\
\hline $\begin{array}{ll}\text { Comorbilidades, } \mathrm{n}(\%) \\
- & \text { Diabetes } \\
- & \text { Hipertensión arterial } \\
- & \text { EPOC } \\
- & \text { Asma } \\
- & \text { Obesidad } \\
- & \text { Enfermedad coronaria } \\
- & \text { Inmunosupresión }\end{array}$ & $\begin{array}{c}14(18,7) \\
18(24) \\
4(5,3) \\
3(4) \\
10(13,3) \\
0(0) \\
3(4)\end{array}$ & $\begin{array}{c}15(19,2) \\
20(25,6) \\
2(2,6) \\
1(1,28) \\
8(10,3) \\
2(2,6) \\
2(2,56)\end{array}$ & $\begin{array}{l}0,929 \\
0,814 \\
0,378 \\
0,292 \\
0,555 \\
0,163 \\
0,332\end{array}$ \\
\hline $\begin{array}{ll}\text { Presentación al ingreso, } \mathrm{n}(\%) \\
- & \text { Asintomática } \\
- & \text { Leve } \\
- & \text { Moderada } \\
- & \text { Grave }\end{array}$ & $\begin{array}{c}4(5,3) \\
22(29,3) \\
34(45,3) \\
15(20)\end{array}$ & $\begin{array}{c}3(3,9) \\
9(11,5) \\
40(51,3) \\
26(33,3)\end{array}$ & 0,030 \\
\hline Duración de la internación, mediana (RIC) & $10(7-12)$ & $7(6-11)$ & 0,142 \\
\hline Pasa a UCC*, n (\%) & $6(8)$ & $9(11,7)$ & 0,444 \\
\hline Mortalidad hospitalaria, n (\%) & $6(8)$ & $8(10,7)$ & 0,575 \\
\hline
\end{tabular}


Se realizó radiografía de tórax en 44 pacientes $(28,7 \%)$ y tomografía en 109 (71,3\%), que mostraron infiltrados intersticiales bilaterales en 95, infiltrados focales en 24 y ausencia de infiltrados en 34.

Luego de la implementación del PROA-COVID se evidenció una disminución significativa en la prevalencia de uso de ATB: $48(64 \%)$ en el P1 versus $32(41 \%)$ en el P2 $(p=0,004)$ (Tabla 2). Se observó un aumento del porcentaje de uso adecuado de antibióticos en $\mathrm{P} 2$ (46,8\% vs $37,5 \%$ ), pero dicha diferencia no alcanzó significación estadística. Entre las causas de tratamiento inadecuado, la indicación innecesaria fue mayor en $\mathrm{P} 1(80 \%$ vs $50 \%, p=0,03)$ y la duración del tratamiento en el P2 (43,7\% vs $13,3 \%, p=0,02)$.

Al ajustar por severidad de la enfermedad no se observaron diferencias significativas en la mortalidad, en la frecuencia de pase a UCC ni en el uso de tratamiento combinado entre ambos períodos (Tabla 3 ).

En los dos períodos se indicaron 80 tratamientos antibióticos (Tabla 4). Veintiocho (35\%) pacientes recibieron tratamiento combinado con macrólidos, 15 con claritromicina y 13 con azitromicina. El tratamiento combinado con macrólidos fue más frecuente en el $\mathrm{P} 2$, asociado a la mayor prevalencia de pacientes con enfermedad grave en dicho período. Se observó una disminución significativa en la prescripción de azitromicina en el P2.
Seevaluóel consumomensual deATB(Figura 1), observándose una disminución del consumo de betalactámicos + inhibidores de betalactamasas (BLIBL) y azitromicina. No se observó un descenso en el consumo de ceftriaxona.

Si tenemos en cuenta exclusivamente el gasto en ATB, sin contemplar otros insumos y costos relacionados, se estima que la reducción del consumo de ATB innecesarios y el acortamiento de la duración de los tratamientos luego de la aplicación del PROA permitirían un ahorro en los costos de $\$ 210.455,17$ en los tres meses posteriores a la intervención, de mantenerse el mismo porcentaje de ocupación de camas.

\section{Discusión}

La pandemia de COVID-19 representa un gran desafío para el sistema de salud y genera un nuevo escenario y nuevos desafíos para los PROA, entre los cuales se encuentran la reasignación de recursos en la planificación de la pandemia, las dificultades generadas por el aislamiento social en recorridas de sala y en la realización de auditorías prospectivas, actividades educativas y reuniones de los comités.

En este contexto particular, la prevalencia de uso de ATB en pacientes con COVID-19 llega hasta el $70 \%$ a $90 \%$ en diferentes estudios, a pesar de la baja prevalencia de coinfección bacteriana reportada. En nuestro hospital, la prevalencia de uso de ATB previa a la implementación del PROA fue del 64\%, muy cercana a las reportadas.

\section{Tabla 2. Uso de antibióticos}

\begin{tabular}{|c|c|c|c|}
\hline & $\begin{array}{c}\text { P1 } \\
n=75\end{array}$ & $\begin{array}{c}P 2 \\
n=78\end{array}$ & p-valor \\
\hline Uso de ATB, n (\%) & $48(64)$ & $32(41)$ & 0,004 \\
\hline Uso adecuado de ATB, $n(\%)$ & $18(37,5)$ & $15(46,8)$ & 0,404 \\
\hline $\begin{array}{l}\text { Causas de inadecuación del tratamiento ATB } \\
-\quad \text { No indicación } \\
-\quad \text { Duración } \\
-\quad \text { Espectro }\end{array}$ & $\begin{array}{c}24(80) \\
4(13,3) \\
2(6,7)\end{array}$ & $\begin{array}{l}8(50) \\
7(43,8) \\
1(6,2)\end{array}$ & $\begin{array}{l}0,035 \\
0,021\end{array}$ \\
\hline Tratamiento combinado con macrólidos, n (\%) & $13(27,1)$ & $15(46,9)$ & 0,069 \\
\hline Uso de azitromicina, $\mathrm{n}(\%)$ & $12(25)$ & $1(3,1)$ & 0,009 \\
\hline
\end{tabular}

\section{Tabla 3. Asociación entre variables de resultado y período evaluado}

\begin{tabular}{|l|c|c|c|}
\multicolumn{1}{|c|}{ Variable de resultado } & OR P2/P1* & IC95\% & p-valor \\
\hline Mortalidad $(n=112)$ & 0,93 & $(0,29-2,98)$ & 0,90 \\
\hline Pase a UCC** $(n=114)$ & 0,96 & $(0,29-3,15)$ & 0,94 \\
\hline Tratamiento ATB combinado $(n=76)$ & 1,38 & $(0,49-3,93)$ & 0,54
\end{tabular}

*Todas las OR fueron ajustadas por severidad de la enfermedad

**UCC: Unidades de cuidados críticos 


\begin{tabular}{|c|c|}
\hline ATB & n (\%) \\
\hline Ceftriaxona & $46(57,5)$ \\
\hline Betalactámico + IBL & $29(36,25)$ \\
\hline Levofloxacina & $3(3,73)$ \\
\hline Piperacilina/tazobactam & $2(2,5)$ \\
\hline
\end{tabular}

Si bien por el momento hay limitados datos de los PROA en la pandemia de COVID-19, existen innumerables estudios que demuestran su impacto previo a la misma. Un metaanálisis publicado en 2016 (15) que evaluó 145 estudios demostró una reducción del riesgo relativo en la mortalidad al prescribir antimicrobianos empíricos dirigidos por guías de recomendaciones, sin mostrar aumento de la mortalidad con el pasaje endovenoso a vía oral, el desescalamiento o la restricción de determinados antimicrobianos.

En nuestra experiencia, la implementación temprana del PROACOVID permitió reducir significativamente la prevalencia de uso de ATB en los pacientes internados, así como mejorar la adecuación de su indicación, especialmente en el uso innecesario y en la indicación no justificada de azitromicina. Si bien la duración inadecuada del tratamiento fue mayor luego del PROA, esto podría deberse a la mayor proporción de pacientes con enfermedad severa en dicho período. Se ha observado que la prescripción de ATB está condicionada por patrones culturales y de comportamiento que influyen a la hora de tomar decisiones (15-17), y puede ser la causa para la continuación de tratamientos ATB más allá del tiempo indicado en pacientes que presentan enfermedad severa.

Un estudio de intervención realizado en nuestro país, recientemente presentado en el ECCVID (ESCMID Conference on Coronavirus Disease), consistente en aplicar un PROA dirigido a disminuir el uso de ceftriaxona en pacientes con COVID-19, demostró una disminución significativa en su consumo luego de su implementación (18).

En nuestro estudio, luego de ajustar por la severidad de la presentación no se observaron cambios en la mortalidad ni en el porcentaje de pase a UCC luego de la implementación del programa, lo cual refuerza la utilidad de la aplicación del PROA, sin que esto impacte en forma desfavorable en el pronóstico de los pacientes.

Otro estudio realizado en Argentina mostró que la implementación progresiva en todo el hospital de un PROA logró reducir significativamente el consumo de ATB y generó un ahorro de USD 913.236 en tres años, además de disminuir la incidencia de infecciones por $P$. aeruginosa del 19\% al $0 \%$ $(p=0,02)$; Staphylococcus aureus resistente a la meticilina de $47 \%$ a $30 \%(p=0,04)$; $P$. mirabilis del $40 \%$ al $27 \%(p=0,05)$; Enterobacter cloacae del $50 \%$ al $10 \%(p=0,03)(19)$.

\section{Figura 1. Consumo de ATB en DDD cada 100 pacientes días. Mayo a septiembre}

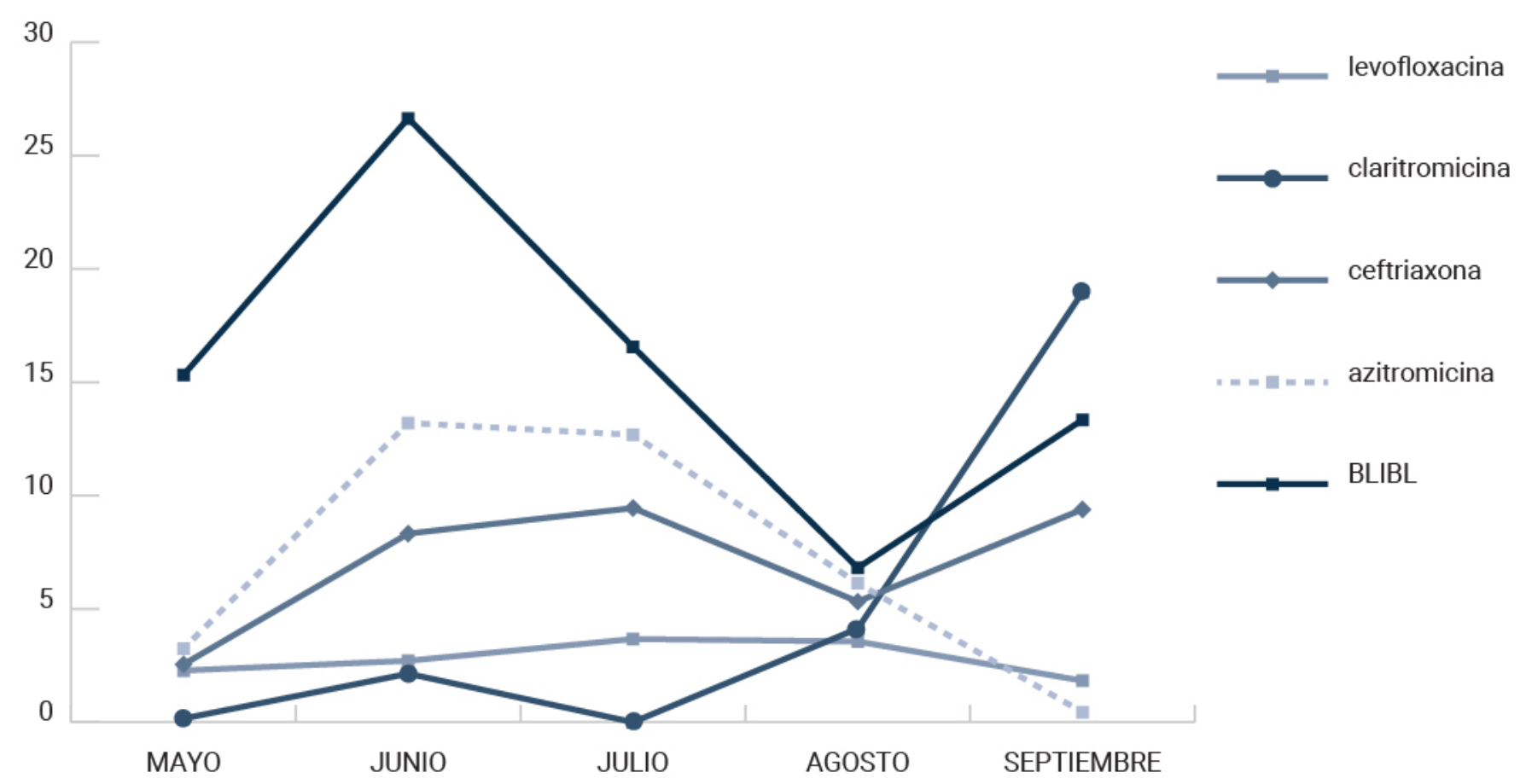


Luego de nuestra intervención se observó una disminución del consumo global de ATB, en especial de BLIBL y azitromicina, a pesar de la mayor severidad de los pacientes en el período posterior al programa. Si bien se evidenció un descenso inicial del consumo de ceftriaxona, este no fue sostenido en el tiempo, probablemente debido a la mayor prevalencia de pacientes con enfermedad severa y a que es un es un ATB que, al administrarse una vez por día, permite menor contacto entre el personal de salud y los pacientes con COVID-19.

Esta disminución en el consumo se asoció a una reducción de los costos hospitalarios, estimándose un ahorro de $\$ 210.455,17$ en un período de tres meses solo en el gasto en ATB. Como limitaciones del estudio no podemos saber si estos cambios serán sostenibles en el tiempo, ya que solo se realizó una primera evaluación posterior a la implementación del PROA. Debido a la falta de grupo control y a que solo se realizaron dos mediciones, no podemos asegurar que los resultados obtenidos se deban exclusivamente a la intervención, y que no hayan sido influidos por otros factores.

Nuestro estudio no tuvo como objetivo evaluar la prevalencia de infecciones bacterianas secundarias ni de OMR, lo cual requiere un mayor seguimiento en el tiempo y podría ser objeto de otro estudio en el futuro cercano.

Debido a que el seguimiento se realizó hasta el alta hospitalaria tampoco pudimos evaluar la evolución de los pacientes a largo plazo.

Como conclusión, la implementación del PROA-COVID fue una estrategia efectiva para reducir el uso de antibióticos y optimizar sus indicaciones, lo que destaca la importancia de estas estrategias aplicadas en forma rápida y oportuna. Mientras la pandemia de COVID-19 continúe es importante dirigir los esfuerzos a la optimización del uso de antimicrobianos para prevenir su empleo innecesario y el desarrollo de resistencia antimicrobiana.

\section{Reconocimientos}

Servicio de Clínica Médica. Hospital General de Agudos Enrique Tornú. Dra. Gabriela Varela. Dr. Darío Leff. 


\section{Bibliografía}

1. Joseph C, Togawa Y, Shindo N. Bacterial and viral infections associated with influenza. Influenza Other Respi Viruses. 2013;7:105-13.

2. Zahariadis G, Gooley TA, Ryall P, Hutchinson C, Latchford MI, Fearon MA, et al. Risk of ruling out severe acute respiratory syndrome by ruling in another diagnosis: Variable incidence of atypical bacteria coinfection based on diagnostic assays. Can Respir J. 2006;13(1):17-22.

3. Assiri A, Al-Tawfiq JA, Al-Rabeeah AA, Al-Rabiah FA, Al-Hajjar S, Al-Barrak A, et al. Epidemiological, demographic, and clinical characteristics of 47 cases of Middle East respiratory syndrome coronavirus disease from Saudi Arabia: A descriptive study. Lancet Infect Dis. 2013 Sep 1;13(9):752-61.

4. Lansbury L, Lim B, Baskaran V, Lim WS. Co-infections in people with COVID-19: a systematic review and meta-analysis. J Infect . 2020 Aug 1;81(2):266-75.

5. Rawson TM, Zhu N, Ranganathan N, Gilchrist M, Satta G, Cooke G, et al. Antibiotics and COVID19. Clin Infect Dis. 2020 May 2.

6. Rawson TM, Ming D, Ahmad R, Moore LSP, Holmes $\mathrm{AH}$. Antimicrobial use, drug-resistant infections and COVID-19. Nat Rev Microbiol. 2020;18(8):409-10.

7. Tiri B, Sensi E, Marsiliani V, Cantarini M, Priante G, Vernelli C, et al. Antimicrobial Stewardship Program, COVID-19, and Infection Control: Spread of Carbapenem-Resistant Klebsiella Pneumoniae Colonization in ICU COVID-19 Patients. What Did Not Work? J Clin Med. 2020;9(9):2744.

8. Rossato L, Negrão FJ, Simionatto S. Could the COVID-19 pandemic aggravate antimicrobial resistance? American Journal of Infection Control; 2020 Sept; 48 (9):1129-30.

9. Cantón R, Gijón D, Ruiz-Garbajosa P. Antimicrobial resistance in ICUs: an update in the light of the COVID-19 pandemic. Current opinion in critical care 2020 Oct;26 (5):433-41.

10. COVID-19 rapid guideline: antibiotics for pneumonia in adults in hospital. NICE guideline Published: 1 May; 2020. https://www.nice.org.uk/

11. Clinical management of COVID-19. https://www. who.int/publications/i/item/clinical-management-ofcovid-19. Accessed September 10, 2020

12. Rawson TM, Moore LSP, Castro-Sanchez E, Charani E, Davies F, Satta G, et al. COVID-19 and the potential long-term impact on antimicrobial resistance. J Antimicrob Chemother. 2020;75(7):1681-4.
13. Huttner BD, Catho G, Pano-Pardo JR, Pulcini C, Schouten J. COVID-19: don't neglect antimicrobial stewardship principles! Clinical Microbiology and Infection 2020; Jul 26(7):808-10.

14. Management of COVID-19 | Coronavirus Disease COVID-19. https://www.covid19treatmentguidelines. nih.gov/overview/management-of-covid-19/. Accessed September 10,2020.

15. Schuts EC, Hulscher MEJL, Mouton JW, Verduin CM, Stuart JWTC, Overdiek HWPM, et al. Current evidence on hospital antimicrobial stewardship objectives: A systematic review and meta-analysis. Lancet Infect Dis. 2016 Jul;16(7):847-56.

16. Gaygısız Ü, Lajunen T, Gaygısız E. Socio-economic factors, cultural values, national personality and antibioticsuse:A cross-cultural studyamong European countries. J Infect Public Health 2017;10(6):755-60.

17. Deschepper R, Grigoryan L, Lundborg CS, Hofstede G, Cohen J, Kelen G Van Der, et al. Are cultural dimensions relevant for explaining cross-national differences in antibiotic use in Europe? BMC Health Serv Res. 2008;8:123.

18. Favier $\mathrm{P}$, Moncalero SA, Abusamra L, D’amico N, Cabrini M, Pérez Blanco LE et al. Implemantation of an antibiotic stewardship progam targeting ceftriaxone use in COVID-19 patients: A fast response in de middle of the pandemic. ESCMID Conference on Coronavirus Disease. September 23-25, 2020 [abstract 314].

19. Bantar C, Sartori B, Vesco E, Heft C, Saúl M, Salamone F, et al. A hospitalwide intervention program to optimize the quality of antibiotic use: Impact on prescribing practice, antibiotic consumption, cost savings, and bacterial resistance. Clin Infect Dis. 2003;37(2):180-6. 
Evaluation of an antimicrobial stewardship program implementation during the global outbreak of coronavirus desease (COVID-19)

Introduction: Despite the low frequency of Covid-19-associated bacterial infections, empirical antibacterial treatment is as high as 70 to $90 \%$. The primary goal of this study was to determine the impact of the implementation of an antimicrobial stewardship program to target Covid-19 patients (ASP-COVID).

Materials and methods: Retrospective, descriptive, and analytic pre and post intervention study. Prevalence and adequacy of antibacterial treatment in hospitalized patients prior and after ASP-COVID implementation were assessed. Monthly antibiotic consumption in $\mathrm{DDD} / 100$ patients-day and costs related with inadequate usage were estimated.

Results: One hundred and fifty three patients, 75 prior and 78 after ASP-COVID implementation, were included. No significant difference in population characteristics between both periods was observed. Moderate and severe clinical presentations were more frequent after the intervention $(p=0,03)$. ASP implementation showed a significant reduction of antimicrobial treatment ( $64 \%$ vs $41 \%, p=0,004)$, with an increment of adequate usage ( $37,5 \%$ vs $46,8 \%, p=N S)$. Unnecessary usage was higher prior to ASP ( $80 \%$ vs $50 \%$, $p$ $=0,03)$ and length of treatment post intervention $(13,3 \%$ vs $43,7 \% \%, p=0,02)$. Program implementation decreased beta lactam antibiotics + IBL and azithromycin consumption.

After adjusting for severity, no significant difference was found in mortality, incidence of ICU admission nor combined antibacterial therapy with macrolides between both periods.

Conclusions: ASP-COVID implementation was an effective strategy in reducing antimicrobial usage and in optimizing antibacterial treatment indications, highlighting the importance of its rapid and timely application.

Keywords: antimicrobial stewardship program, COVID-19, antibiotics. 